\title{
A Pilot Study Evaluating the Physiological Parameters of Performance-Induced Stress in Undergraduate Music Students
}

\author{
Esther M. van Fenema ${ }^{a}$ Pim Gal $^{b}$ Maxime V. van de Griend ${ }^{a, b}$ \\ Gabriel E. Jacobs ${ }^{a}$ b Adam F. Cohen ${ }^{b, c}$ \\ a Department of Psychiatry, Leiden University Medical Center, Leiden, The Netherlands; \\ ${ }^{b}$ Centre for Human Drug Research, Leiden, The Netherlands; ' ${ }^{\mathrm{C}}$ Department of Nephrology, \\ Leiden University Medical Center, Leiden, The Netherlands
}

\section{Keywords}

Activity monitors · Digital biomarkers · Highly wearable biosensing system · Wearables

\begin{abstract}
Music performance anxiety (MPA) is a specific condition for musicians. Although it can have a negative influence on their music careers, little attention is paid to this phenomenon both in the professional environment and in stress research. In the current pilot study, insight was gained into the physiology of the autonomic stress response related to anxiety in musicians when performing on stage by using a wearable biosensor patch for registration of a range of physiological parameters. Also, the validity of two different psychometric questionnaires in objectifying the stress response on stage to predict the individual stress response was explored. The autonomic physiological parameters (heart rate, respiratory rate, skin temperature) of 11 violists and violinists were collected while performing on stage and in resting state using the VitalConnect HealthPatch ${ }^{\circledR}$. In addition, scores on validated questionnaires in research on MPA (State Anxiety Inventory, Kenny Music Performance Anxiety Inventory, Short Form Health Survey) were collected in order to try to objectify the magnitude of the subjective level of both MPA and experienced stress. The registration of the autonomic parameters showed a significant increase in heart rate, respiratory rate, and stress level from resting state measurements during stage performance. Analysis of heart rate variability showed a shift from indices of parasympathetic nervous system activity during baseline measurements towards indices of sympathetic nervous system activity during stress measurements. Surprisingly, none of the questionnaires was correlated to the physiological stress parameters on stage. In conclusion, the wearable biosensor patch proved to be an adequate tool to assess physiological stress parameters on stage. The different questionnaires did not contribute to the prediction of its occurrence in a group of musicians.


van Fenema et al.: Physiological Parameters of Performance-Induced Stress

\section{Introduction}

Performance-induced stress, also referred to as "music performance anxiety" (MPA) or "stage fright," is part of the daily routine for many musicians and can be an invalidating problem; it is characterized not only by psychological symptoms, but also by specific physical symptoms such as sweating, heart palpitations, and tremor [1-6]. The physiological response to stress is mediated by both activation of the autonomic nervous system (ANS) and the hypothalamic-pituitary-adrenal axis [7]. Results of studies on the physiological response to stress showed that also musicians performing on stage in front of an audience were particularly prone to increased stress-related physical parameters, such as heart rate, respiratory rate, perspiration, and muscle tension [6, 8-13]. The Yerkes-Dodson law describes the functional nature of this stress response: when the arousal rises, performance quality improves until a certain level of arousal is reached. Very low levels of arousal are thought to give rise to performances of lesser quality, whereas excessive arousal can interfere with the performance because of loss of steadiness in the hands and memory blocks [14]. Therefore, although a certain level of stress activation can be beneficial to musicians, an exaggerated stress response is expected to negatively influence their performance.

Not all people respond in the same way to stressful circumstances. It has been demonstrated that certain individuals are more vulnerable to the physical and psychological impact of stress than others [15-17]. Since MPA is an important challenge for musicians during their careers, in the current study we aimed to explore the link between the physiological markers of stress and the psychological components of MPA.

However, limited data on the temporal behavior of the physiological stress-induced response are available. Therefore, the aim of the present study was to characterize the temporal physiological stress response in conservatory performers and to evaluate the difference with physiological parameters in the resting state. In addition, the correlation between physiological stress parameters and validated questionnaires in MPA research was investigated.

\section{Methods}

\section{Participants}

Eleven viola and violin players were recruited from the Amsterdam Conservatory in the Netherlands. Only players of these instruments were included in order to create a homogenous group of musicians for this pilot study. Violinists and violists were considered suitable subjects since they represent a relatively large homogeneous group of musicians. Students using anxiolytics or drugs affecting the cardiovascular system were not included in this study. Participants were enrolled between March and May of 2016.

\section{Physiological Measures}

The VitalConnect HealthPatch ${ }^{\circledR}$ (VitalConnect, Campbell, CA, USA) is an oblong adhesive biosensor patch containing two ECG electrodes, a thermistor, and an accelerometer. The patch is placed on the left side of the chest underneath the pectoralis major muscle, the reason for this being the position of the violin on the left shoulder and the preferred invisibility of the patch during performance on stage. The biosensor patch was used to obtain heart rate (automatically via single-lead ECG), respiratory rate (algorithm with ECG and accelerometer), skin temperature, and skin conductance (between electrodes). Figure 1 displays the patch that was used for this study. 
Fig. 1. VitalConnect HealthPatch. The VitalConnect HealthPatch was used in this study to record a single-lead ECG, respiratory rate, and temperature. The patch was positioned just caudal of the left pectoral muscle.

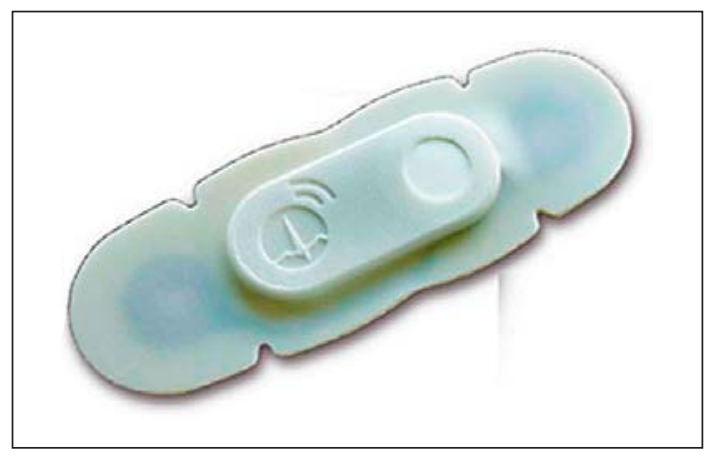

Heart rate variability (HRV) is the physiological phenomenon of variation in the time interval between heartbeats, caused by several factors including the baroreceptor feedback loop. The change in heart rate induced by the parasympathetic and the orthosympathetic nervous system can be differentiated by frequency domain analysis [18]. HRV in the present analysis was derived from the single-lead ECG, and the area under the curve was calculated for very-low-frequency HRV (VLF-HRV, <0.04 Hz), low-frequency HRV (LF-HRV, 0.04$0.15 \mathrm{~Hz}$ ), and high-frequency HRV (HF-HRV, $0.15-0.4 \mathrm{~Hz}$ ). HF-HRV is considered a marker of parasympathetic nervous system tone, whereas VLF-HRV and LF-HRV are considered markers for a combination of the orthosympathetic and parasympathetic ANS tone.

Several papers published by the manufacturer showed that the wireless patch records data in a robust and reliable way $[19,20]$. The data recorded by the wireless patch were directly and continuously transferred to a connected iPod for the entire duration of the recording.

\section{Psychological Questionnaires}

The Short Form Health Survey (SF-36) was used to get a general view of all participants' health. The SF-36 consists of 36 items on eight domains: physical functioning, social functioning, mental health, perceived general health, bodily pain, vitality, limitations due to physical health problems, and emotional problems. A higher score indicates a higher level of well-being [21].

The Kenny Music Performance Anxiety Inventory (K-MPAI) was used to assess the subject's experience of MPA [22]. The questionnaire consists of 26 theses addressing MPA by measuring the cognitive, physiological, and behavioral components of performance anxiety [23]. A higher score indicates greater psychological distress and anxiety [17], although the questionnaire has not been fully validated.

The Spielberger State-Trait Anxiety Inventory (STAI) was filled in by the participants 30 min before they performed on stage in front of an audience. The STAI consists of two separate questionnaires: the first questionnaire regards how someone feels at that exact moment (state anxiety, STAI-DY1), and the second regards how someone feels in general (trait anxiety, STAI-DY2). In the current study we only used the STAI-DY1 to indicate the perceived level of anxiety directly prior to the music performance. In this manner we tried to capture the feelings of stress or anxiety the students perceived just before getting on stage.

\section{Baseline Recording}

The study was conducted at the Amsterdam Conservatory and consisted of two sessions. During the first session, baseline measurements of the physiological parameters were recorded during an period of $5 \mathrm{~min}$ of a 1-h recording of the wireless patch. The subjects were seated and in a resting state. During this hour, the subjects completed the K-MPAI and SF-36 questionnaires. 
Table 1. Baseline characteristics of the study subjects $(n=11)$

$\begin{array}{lc}\text { Age, years } & 22.5 \pm 3.0 \\ \text { Male sex } & 3(27 \%) \\ \text { Body mass index } & 21.15 \pm 2.2 \\ \text { Exercise, h/week } & 4.2 \pm 2.6 \\ \text { SF-36 Emotional Problems } & 4.6 \pm 1.3 \\ \text { SF-36 Emotional Well-Being } & 19.9 \pm 3.7 \\ \text { SF-36 Social Functioning } & 7.9 \pm 1.8 \\ \text { Kenny Music Performance Anxiety Inventory } & -17.8 \pm 20.3 \\ \text { State Anxiety Inventory } & 41.8 \pm 6.5 \\ \text { Drug use in last 12 h before study recording } & \text { none }\end{array}$

Performance Recording

The second recording session was a performance at the conservatory during which the students played their instrument, either alone or with piano accompaniment, on stage in front of an audience. These performances were a monthly recurring event arranged by the teachers for all music students at the Amsterdam Conservatory. These performances were regarded by the students as being very stressful events, since their performance was crucial in the tutor's decision which subjects could continue to the next course. The wireless patch was applied on the chest at least $30 \mathrm{~min}$ before the start of the performance (whenever there was an appropriate time window), after which all subjects completed the STAI-DY1. The wireless patch was worn by the subjects during the entire performance and was removed shortly thereafter.

\section{Statistical Methods}

Data were analyzed using the IBM SPSS Statistics 24 statistical software (Chicago, IL, USA). Data are presented as mean \pm SD or median with interquartile range where appropriate. A paired-sample $t$ test was used to compare continuous variables. Continuous variables were correlated with a Pearson correlation coefficient, and the K-MPAI and STAI-DY1 questionnaires were correlated with an intraclass correlation coefficient (ICC). No formal power analysis was performed because this was a pilot study to evaluate the feasibility of assessment of stage-induced physiological parameters with a wearable device. The level of significance was set at $p<0.05$.

\section{Results}

\section{Sociodemographic Data}

Eleven subjects were included in this pilot study. Their baseline characteristics are displayed in Table 1. Our sample included healthy students, mainly female and with normal values for the SF-36 questionnaire. Table 2 displays the difference between the baseline and stress measurements. Heart rate and respiratory rate were significantly different between the two settings. Similarly, VLF-HRV relative power was significantly increased, whereas LF-HRV relative power was significantly reduced.

\section{Temporal Effect of Stress}

Heart rate and VLF-HRV relative power were already increased compared to baseline at the start of the performance recording, up to $60 \mathrm{~min}$ before the start of the performance. The maximum increase in both parameters was achieved during the performance. The decline in both parameters was initiated immediately at the end of the performance and returned to 
Table 2. Means, standard deviations, and Wilcoxon rank tests of physiological parameters and heart rate variability during resting state and stress measurements

\begin{tabular}{llcc}
\hline & Resting state & Performance & $p$ value \\
\hline Heart rate, beats/min & $74.8 \pm 9.6$ & $137.7 \pm 17.0$ & $<0.0001$ \\
Respiratory rate, breaths/min & $15.5 \pm 3.0$ & $20.4 \pm 2.6$ & 0.004 \\
VLF-HRV relative power, \% & $38.6 \pm 16.8$ & $71.3 \pm 20.9$ & 0.007 \\
LF-HRV relative power, \% & $39.9 \pm 14.1$ & $20.0 \pm 11.5$ & 0.009 \\
HF-HRV relative power, \% & $21.5 \pm 13.4$ & $8.7 \pm 13.3$ & 0.065 \\
\hline
\end{tabular}

HF-HRV, high-frequency heart rate variability; LF-HRV, low-frequency heart rate variability; VLF-HRV, very-low-frequency heart rate variability.

Fig. 2. Example of heart rate, respiratory rate, and VLF-HRV fraction change surrounding the performance. This figure displays the change in heart rate (a), respiratory rate (a), and VLF-HRV (b) before, during, and after the performance in one of the subjects. a A clear increase in heart rate and respiratory rate can be observed, starting about $20 \mathrm{~min}$ before the start of the performance until just after the end of the performance (grey shading), quickly dropping after the end of the performance. b A similar pattern can be observed in VLF-HRV, which increases from $20 \mathrm{~min}$ before the performance, peaks during the performance (red bar), and quickly drops back to its baseline level after the performance. bpm, beats per minute; $H R$, heart rate; RR, respiratory rate; VLF-HRV, verylow-frequency heart rate variability.
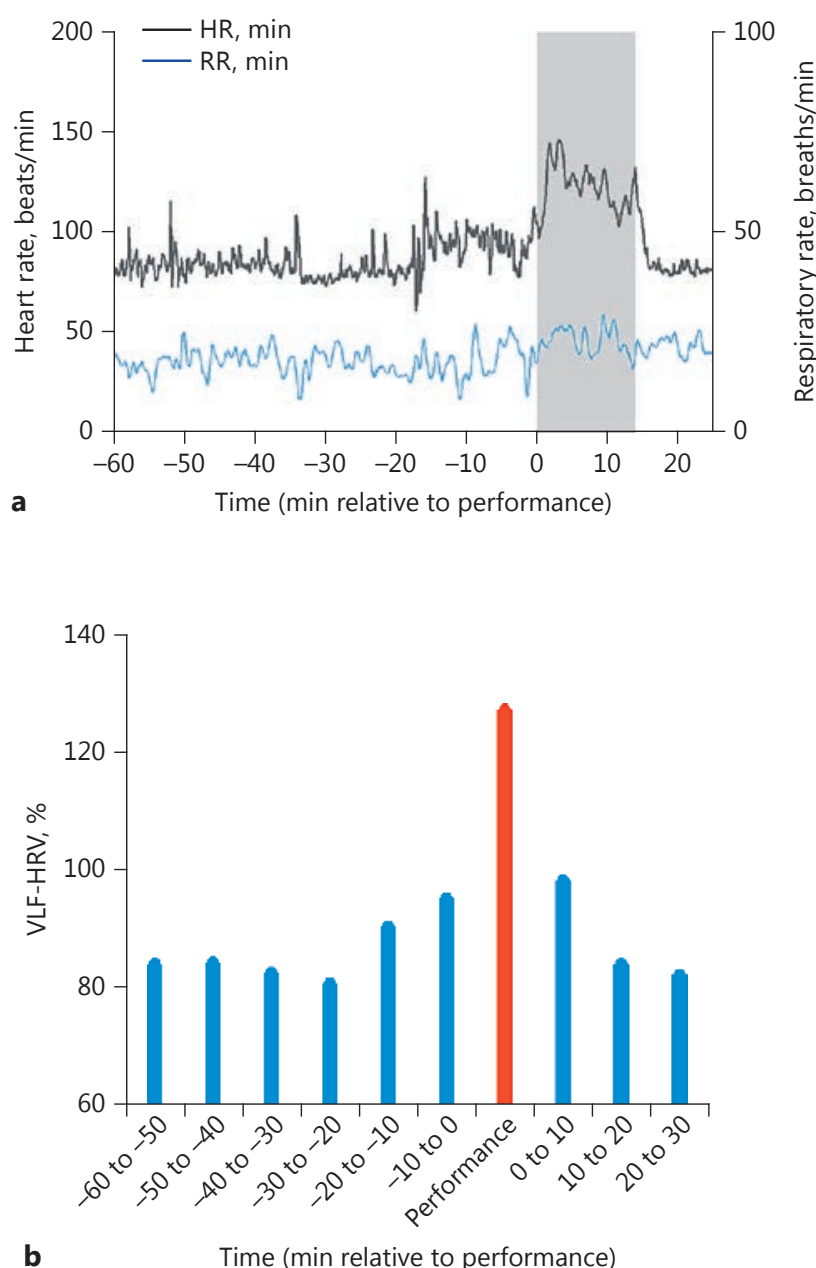

baseline values about 20 min later. Heart rate and VLF-HRV relative power during the performance showed a strong correlation (ICC $=0.729, p=0.004$ ). Figure 2 displays the heart rate, respiratory rate, and VLF-HRV relative fraction for one of the subjects and displays the heart rate and VLF-HRV relative power during the performance. 


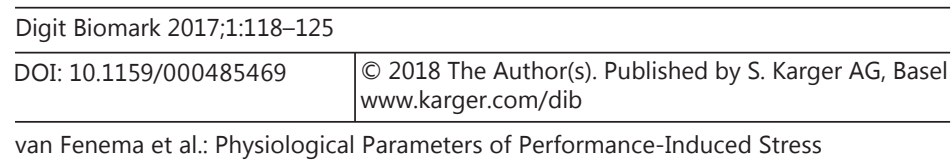

Table 3. Correlation between questionnaires and physiological parameters

\begin{tabular}{lrrrr}
\hline & K-MPAI & $p$ value & STAI & $p$ value \\
\hline Heart rate during performance & 0.053 & 0.884 & -0.119 & 0.743 \\
Respiratory rate during performance & 0.211 & 0.616 & -0.162 & 0.702 \\
VLF-HRV relative power during performance & 0.111 & 0.760 & -0.573 & 0.083 \\
LF-HRV relative power during performance & 0.003 & 0.994 & 0.554 & 0.097 \\
HF-HRV relative power during performance & -0.175 & 0.629 & 0.435 & 0.210 \\
\hline
\end{tabular}

HF-HRV, high-frequency heart rate variability; K-MPAI, Kenny Music Performance Anxiety Inventory; LF-HRV, low-frequency heart rate variability; STAI, State-Trait Anxiety Inventory; VLF-HRV, very-lowfrequency heart rate variability.

\section{Self-Report Questionnaires}

There was no correlation between the K-MPAI and STAI questionnaires (ICC $=0.038, p=$ 0.459 ) (Table 3). None of the physiological parameters, including heart rate and respiratory rate, was significantly correlated to the questionnaire results, although the correlation coefficient between the STAI questionnaire and VLF-HRV and LF-HRV exceeded 0.5.

\section{Discussion}

The main goal of this pilot study was to gain insight into the physiology of the autonomic stress response in musicians by using a wearable biosensor patch, and to explore the association between the physiological measurements and psychometric questionnaires on state anxiety and MPA. To achieve these goals, we assessed the effects of performance-induced stress on physical parameters in violists and violinists of the Amsterdam Conservatory, explored the association between the physiological variables and psychological endpoints, and investigated the association between subjective levels of experienced stress and objective activation of the ANS.

Comparison of the physiological parameters while performing on stage versus measurements under baseline resting state conditions revealed increased heart rate, increased respiratory rate, and changes in HRV indices associated with an increase in sympathetic tone. The used wireless biosensor patch proved to be a useful tool for quantification of stress on stage. Furthermore, the STAI-DY1 did not correlate significantly with the physiological parameters of the stress measurement. Regarding the K-MPAI questionnaire, no significant correlation was found between the self-report questionnaires and the physiological parameters of the resting state measurement or the stress measurement.

The observed increase in heart rate and respiratory rate during stressful circumstances and increase in indices of sympathetic tone are consistent with the existing literature on the physiology of stress and HRV $[6,8-13,16]$. According to Williamon et al. [12], the highfrequency component of the HRV reflects parasympathetic nervous system activity, whereas the low-frequency component reflects a balance between orthosympathetic and parasympathetic nervous system activity. Stress incudes changes in the balance between the parasympathetic and the sympathetic nervous system in favor of sympathetic activity. The shift in activity of the different components of the ANS resulted in increased biological parameters as detected by the wireless patch, which therefore might have potential to detect biological responses to stress. 
A surprising finding was that the level of perceived anxiety (as assessed with the STAIDY1) did not correlate with the measurements of heart rate, respiratory rate, stress level, and temperature during performance on stage. This could be due to an underestimation of one's subjective feelings of stress/anxiety, since the STAI-DY1 asks the participant to assess his/ her feelings about an upcoming stressful event, or maybe this finding is due to the fact that the music students in our sample are used to performing on stage and experiencing stress. The same applies to the K-MPAI: the scores on this questionnaire did not correlate with either the STAI questionnaire or the measurements of heart rate, respiratory rate, stress level, and temperature during performance on stage. Seen the fact that this list has been produced to detect "stress on stage," it was expected that it would be correlated to the stress measurements. In addition, this questionnaire has not been validated, there are no clear cutoff points, and there has been criticism from musicians that the actual stage experience is insufficiently reflected. Our results support this criticism.

Many different coping strategies used by musicians (including use of sedatives, use of natural substances, alcohol, psychological counselling, and breathing exercises) have been reported in the literature $[1,4,11,24,25]$. The current study registered the effects of performing on stage on body and mind, but we did not ask our participants which coping strategies they used in preparation of a performance. Remarkably, only one of the participants reported the use of beta-blockers for stressful performances or auditions, but did not use beta blockers during the participation in this study. Fishbein et al. [1] reported the use of beta-blockers, either occasionally or prescribed and on a regular basis, by more than onefourth of the 2,122 musicians in their study. However, lower rates of sedative use such as 4.3, 12 , and $17 \%$ have also been reported $[6,24,25]$.

Taken together, the findings of this study offer a promising new method for detecting performance-induced stress in musicians or other performance domains: the use of a wearable biosensor patch which provides real-time information on a number of physiological parameters related to ANS activation.

\section{Statement of Ethics}

All participants gave written informed consent before the onset of the study. For their participation, the students received a voucher of EUR 25. The research protocol synopsis was reviewed by the Medical Ethical Committee of the Leiden University Medical Hospital and considered not subject to the Dutch law of medical scientific research (WMO; Wet Medischwetenschappelijk Onderzoek).

\section{Disclosure Statement}

The authors declare no conflicts of interest.

\section{References}

1 Fishbein M, Middlestadt SE, Ottati V, Straus S, Ellis A: Medical problems among ICSOM musicians: overview of a national survey. Med Probl Perform Art 1988;3:1-8.

-2 van Kemenade JF, van Son MJ, van Heesch NC: Performance anxiety among professional musicians in symphonic orchestras: a self-report study. Psychol Rep 1995;77:555-562.

3 Langendörfer F, Hodapp V, Kreutz G, Bongard S: Personality and performance anxiety among professional orchestra musicians. J Individ Differ 2006;27:162-171. 


\begin{tabular}{l|l}
\hline Digit Biomark 2017;1:118-125 \\
\hline DOI: 10.1159/000485469 & $\begin{array}{l}\text { C } 2018 \text { The Author(s). Published by S. Karger AG, Basel } \\
\text { www.karger.com/dib }\end{array}$ \\
\hline
\end{tabular}

van Fenema et al.: Physiological Parameters of Performance-Induced Stress

-4 Middlestadt SE, Fishbein M: Health and occupational correlates of perceived occupational stress in symphony orchestra musicians. J Occup Med 1988;30:687-692.

5 Studer R, Gomez P, Hildebrandt H, Arial M, Danuser B: Stage fright: its experience as a problem and coping with it. Int Arch Occup Environ Health 2011;84:761-771.

6 Wesner RB, Noyes R, Davis TL: The occurrence of performance anxiety among musicians. J Affect Disord 1990; 18:177-185.

7 McEwen BS: Psychology and neurobiology of stress and adaptation: central role of the brain. Physiol Rev 2007; 87:873-904.

8 Endo S, Juhlberg K, Bradbury A, Wing AM: Interaction between physiological and subjective states predicts the effect of a judging panel on the postures of cellists in performance. Front Psychol 2014;5:773.

-9 Frederikson M, Gunnarsson R: Psychobiology of stage fright: the effect of public performance on neuroendocrine, cardiovascular and subjective reactions. Biol Psychol 1992;33:51-61.

-10 Studer R, Danuser B, Hildebrandt H, Arial M, Gomez P: Hyperventilation complaints in music performance anxiety among classical music students. J Psychosom Res 2011;70:557-564.

-11 Studer R, Danuser B, Wild P, Hildebrandt H, Gomez P: Psychophysiological activation during preparation, performance, and recovery in high- and low-anxious music students. Appl Psychophysiol Biofeedback 2014; 39:45-57.

12 Williamon A, Aufegger L, Wasley D, Looney D, Mandic DP: Complexity of physiological responses decreases in high-stress musical performance. J R Soc Interface 2013;10:20130719.

13 Yoshie M, Kudo K, Murakoshi T, Ohtsuki T: Music performance anxiety in skilled pianists: effects of socialevaluative performance situation on subjective, autonomic, and electromyographic reactions. Exp Brain Res 2009;199:117-126.

14 Parncutt R, McPherson G: The Science and Psychology of Music Performance: Creative Strategies for Teaching and Learning. Oxford, University Press, 2002.

15 Spahn C, Echternach M, Zander M, Voltmer E, Richter B: Music performance anxiety in opera singers. Logoped Phoniatr Vocol 2010;35:175-182.

16 Gouin JP, Deschenes SS, Dugas MJ: Respiratory sinus arrhythmia during worry forecasts stress-related increases in psychological distress. Stress 2014;17:416-422.

17 Rae G, McCambridge K: Correlates of performance anxiety in practical music exams. Psychol Music 2004;32: 432-439.

18 Heart rate variability: standards of measurement, physiological interpretation and clinical use. Task Force of the European Society of Cardiology and the North American Society of Pacing and Electrophysiology. Circulation 1996;93:1043-1065.

19 Chan AM, Selvaraj N, Ferdosi N, Narasimhan R: Wireless patch sensor for remote monitoring of heart rate, respiration, activity, and falls. Conf Proc IEEE Eng Med Biol Soc 2013;2013:6115-6118.

20 Chan AM, Ferdosi N, Narasimhan R: Ambulatory respiratory rate detection using ECG and a triaxial accelerometer. Conf Proc IEEE Eng Med Biol Soc 2013;2013:4058-4061.

21 Aaronson NK, Muller M, Cohen PD, Essink-Bot ML, Fekkes M, Scanderman R, Sprangers MA, te Velde A, Verrips E: Translation, validation, and norming of the Dutch language version of the SF-36 Health Survey in community and chronic disease populations. J Clin Epidemiol 1998;51:1055-1068.

22 Barbar AE, Crippa JA, Osório FL: Parameters for screening music performance anxiety. Rev Bras Psiquiatr 2014;36:245-247.

23 Citroen L, van der Loo M: Podiumangst: uit de schaduw van de coulissen. Psycholoog 2014;April:49-58.

24 Steptoe A, Fidler H: Stage fright in orchestral musicians: a study of cognitive and behavioural strategies in performance anxiety. Br J Psychol 1987;78:241-249.

25 Steptoe A: Stress, coping and stage fright in professional musicians. Psychol Music 1989;17:3-11. 